\title{
Power Allocation and Generator Sizing Optimisation of More-Electric Aircraft On-board Electrical Power during Different Flight Stages
}

\author{
Xin Wang, ${ }^{1}$ Serhiy Bozhko ${ }^{2}$, Jason Atkin ${ }^{3}$, Christopher Hill ${ }^{4}$ \\ University of Nottingham, Nottingham, NG7 2RD, United Kingdom
}

\begin{abstract}
The development of future More Electric Aircraft (MEA) requires exploitation of lightweighted on-board Electrical Power System (EPS) architectural design and energy saving power management strategies. Seeking for the optimal solutions in these key aspects, two vital prerequisites should be ensured for the entire flight stages with load requirements changes one is the optimal power allocation (OPA) aiming to route the power in the system with less transmission losses, and the other one is the optimal generator sizing (OGS) targeting on minimising the required overload capabilities for a lighter generator. Despite different objectives, these two optimisation problems should both meet the load requirements and system operation constraints, including the power balancing and limitations, contactor switching logic, the transmission losses of cables, and the nonlinear converter efficiencies. In this paper, we first propose a formulation method based on the optimal power flow (OPF) model to constitute the system constraints for both optimisation problem, and combining with specific objectives and constraints, the problems of OPA and OGS can be mathematically modelled. By linearizing the nonlinear transmission efficiencies with piecewise functions, the proposed models can be solved using the mixed-integer linear programming (MILP) in CPLEX solver.
\end{abstract}

\section{Nomenclature}

The main symbols used in this paper are defined below. Others will be defined as required in the text.

A. Indices and Numbers

$\begin{array}{ll}i & =\text { Index for generators } \\ j, k & =\text { Index for HV and LV buses respectively } \\ c & =\text { Index for DC/DC converter } \\ p, q & =\text { Index for HV and LV loads respectively } \\ N^{H V B} & =\text { Number of the HV buses } \\ N^{L V B} & =\text { Number of the LV buses } \\ N^{C} & =\text { Number of the DC/DC converters } \\ N^{G} & =\text { Number of the generators }\end{array}$

B. Parameters

$\alpha_{i}^{G} \quad=$ Connection status between the generator $i$ and corresponding HV bus (binary value)

$\eta_{c}, \eta^{\prime}{ }_{c}=$ Efficiency of DC/DC converter in buck/boost mode

$\epsilon_{j}^{A H V}=$ Transmission efficiency in cables between the APU bus and HV bus $j$

$\epsilon_{k k^{\prime}}^{L V} \quad=$ Transmission efficiency in cables of LV bus connection between bus $k$ and $k^{\prime}$

$\epsilon_{j c}^{H V C}=$ Transmission efficiency in cables between the HV bus $j$ and DC/DC converter $c$

\footnotetext{
${ }^{1}$ Marie Skłodowska-Curie Early Stage Researcher \& PhD student, Institute for Aerospace Technology.

${ }^{2}$ Professor, Institute for Aerospace Technology.

${ }^{3}$ Associate Professor, School of Computer Science.

${ }^{4}$ Senior Research Fellow, Institute for Aerospace Technology.
} 

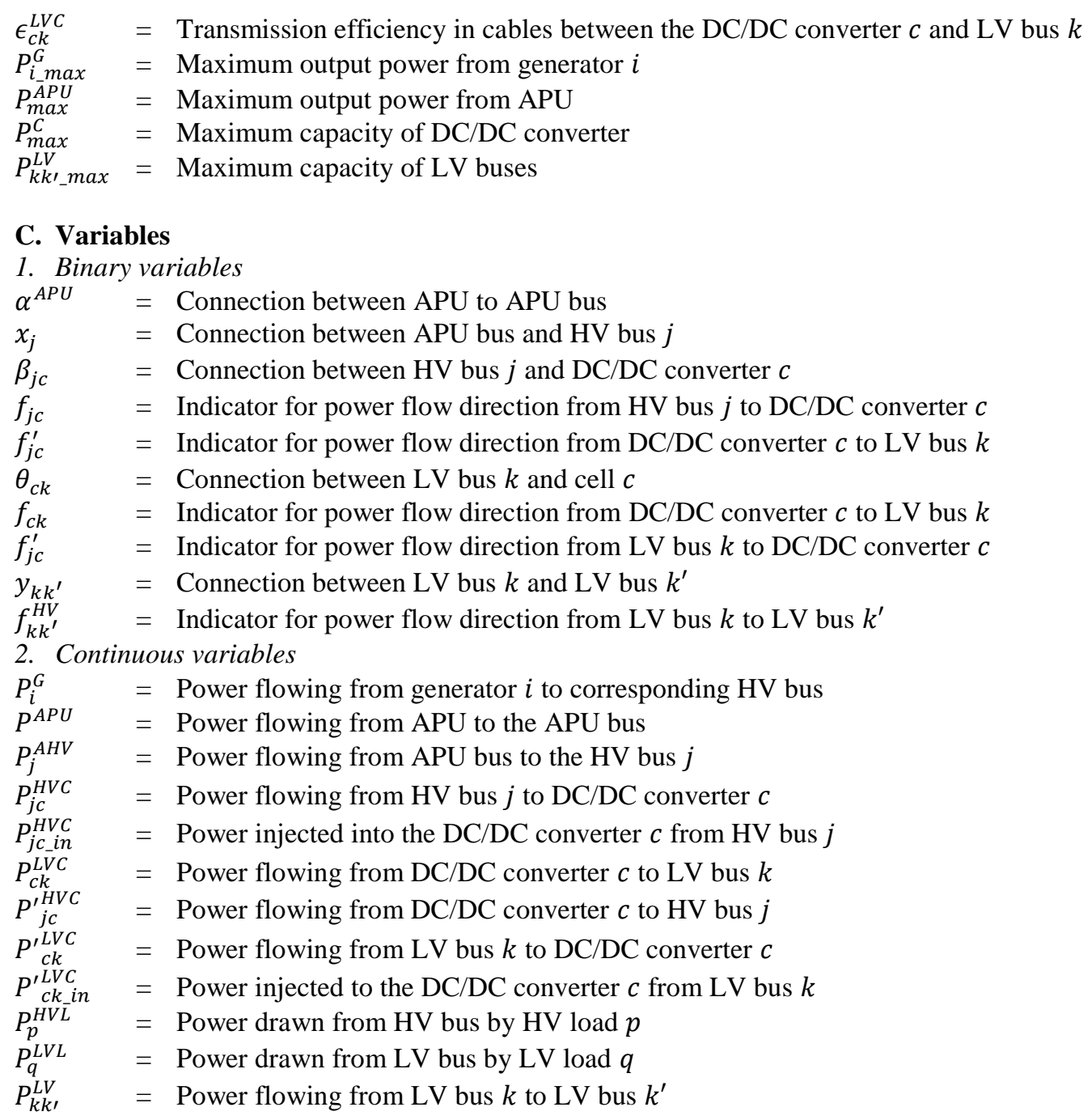

\section{Introduction}

Electrical power demand has been rising significantly in modern aircraft due to more complex on-board electrical/electronic systems, such as In-Flight Entertainment (IFE) and Seat Power Supply (SPS), and the replacement of traditional hydraulics and pneumatics by electrical systems to achieve better dynamic response and higher efficiency [1][2]. Therefore, the concept of More Electric Aircraft (MEA) and All Electric Aircraft (AEA) has been put forward as a main development tendency for future aircraft to make air travel more efficient and environmentally friendly[3]. In the meantime, the High Voltage Direct Current (HVDC) distributed networks are favoured for the MEA for less power losses and weight reduction reasons [4]. This brings great challenges to power allocation and management of the on-board power system because the architecture is getting complex with the adoption of power electronics converters, which enables bidirectional power flow and results in different constraints regarding reliability, priority and efficiency. Besides, reducing the overall weight of the electrical power system (EPS) is also important in the development of aircraft in order to seek better energy efficiency[5]. Optimizing the power distribution intelligently can minimize the required overload capacity needs of the generators, which contributes a lot to reducing the total weight of the power system.

In this paper, we address two main optimisation problems with regard to the aforementioned aspects, one is defined as the optimal power allocation (OPA) problem, aiming to reduce the total power needs from the power sources by scheduling the power in the system with less transmission losses, while the other one is defined as the optimal generator sizing (OGS) problem targeting on minimising the required overload capabilities for each generator. These two problems are investigated for a novel HVDC EPS architecture based on modular power converters (MPC) shown in Fig. 1, where each DC/DC converter can operate in buck/boost mode allowing bidirectional power flow. The 
objective is therefore to formulate a solvable mathematical model for both OPA and OGS problems subjected to the power system operation constraints, including the power balancing and the limitations for each bus/converter, the bus/converter connection logic, the transmission losses in the cables and the nonlinear converter efficiency characteristics. In addition, this is not really a static problem: when the load requirements change during different flight stages, the optimal solutions will change to accommodate the changing loads. This paper proposes a formulation method based on an optimal power flow (OPF) model which is applied to the OPA and OGS problems with modified cost functions and constraints, and provides mathematical models for both problems. The nonlinear functions caused by efficiencies and losses in the models are modelled using a piecewise linearization method to deal with these nonlinear constraints. Using the proposed method, the models can be formulated as Mixed Integer Linear Progam (MILP) models and solved using the CPLEX MILP solver.

Our approach builds on a number of results that opened the way for a more formal EPS optimisation methodology. In [6], the authors present an optimal load management system to route power from the generators to vital loads and design a hierarchical optimal power control strategy, formulating the problem as a MILP model. In [7], the authors formulate the power allocation and scheduling problem by mixed integer quadratic programming (MIQP) to minimise the load shedding and optimal generator operation profiles, and a similar simplified OPA model for a AC/DC hybrid EPS system is proposed in [8] with multi-objectives for bus priority and optimal usage of the storage system. However, none of these studies have considered the converters nor the transmission losses in their models. The power loss during power conversion is considered in power balance equations in [9][10], and in that research, a power management system is designed to control the generators to operate at high efficiency. However, the transmission efficiencies are simplified by treating them as constant values, and the model only considered the generator and HV bus side. The OGS problem has been investigated for terrestrial EPS, for instance, the optimal location and sizing of distributed generation sources are formulated in [11] and [12] to minimize the network power losses and better voltage regulation within the frame work of system security constraints in a redial distribution system. These studies contain load flow analysis in their model and they both focus on AC terrestrial grids, while the OGS problem in MEA proposed in this paper should consider different constraints and modelling methodologies.

The rest of the paper is organized as follows: Section III introduces the studied MPC HVDC EPS architecture. Section IV demonstrates the formulation of OPA and OGS problems based on the OPF model, and Section V explains the linearization methods for the model. Section VI shows the simulation results of the OPA and OGS in different flight stages. Finally, in Section VII, conclusions are drawn.

\section{System Description}

A novel MPC HVDC EPS architecture is shown in Fig. 1, which is comprised of a 28V LVDC network and a 270V HVDC network. The main generators and auxiliary generator power the HV buses via AC/DC rectifiers, and HV loads, such as electric actuators and de-icing systems, consumes power directly from HV buses. The LV buses are connected with the HV buses through bidirectional DC/DC power converters. The LV loads are connected to LV buses, as well as the energy storage system, which is not the focus of this paper.

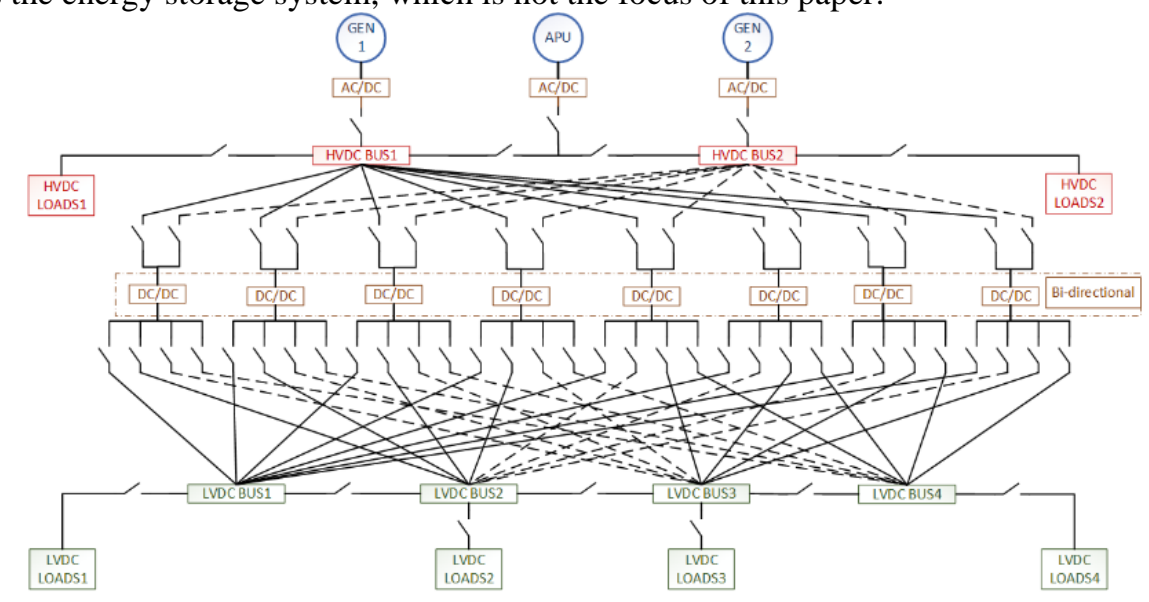

Fig. 1 A novel MPC based HVDC EPS architecture

To concentrate on power flow analysis, a single line power flow diagram, as shown in Fig. 2, is built for the studied HVDC architecture. In this diagram, the devices, such as generators, buses, converters and loads, are represented by nodes, the power flows are given as lines where the arrow indicates flow direction. The unidirectional power flows 
from main generators/APU generator to HV/APU buses, and from the buses to loads, are represented by blue arrow lines. Bidirectional power flows between the buses and converters and among LV buses are represented by red lines.

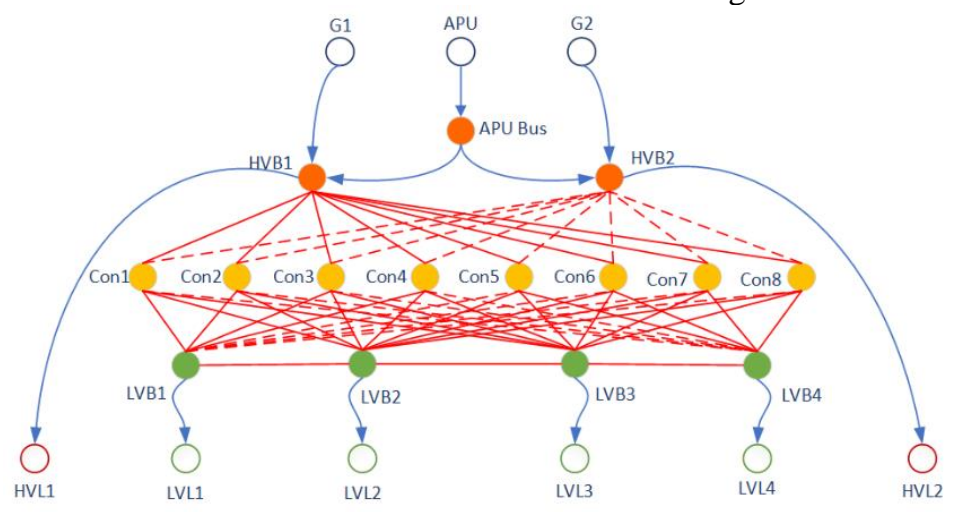

Fig. 2 Simplified power flow diagram of the MPC HVDC EPS

\section{Problem Formulation Based on Optimal Power Flow Model}

The optimal power allocation problem aims to route the power in the system with lower transmission losses, while the optimal generator sizing problem aims to minimise the generator overload requirement, allowing smaller, lighter generators to be used. Despite different objectives, these two optimisation problems should both meet the load requirements and system operation constraints, which can be formulated mathematically based on the optimal power flow (OPF) model to present the power balance, upper/lower bounds of the power for each node, and combined with bus and converter connection constraints. Voltages and currents are not considered so far and the study only refers to the active power flows.

\section{A. Power balance constraints with consideration of power efficiency and bidirectional power flow}

For each bus node, the power flowing through should comply with this rule - the sum of powers flowing into/out each node equals zero (Kirchhoff's Current Law for a given voltage). As for the converter nodes, the power flowing out of each node is less than the power flowing into it because of the converter efficiency. The novel MPC EPS allows bidirectional power flow by operating the converters in either buck or boost mode, and the power flow in each direction is represented by a non-negative decision variable.

For the APU bus node, the power balance equation in (1) represents the power drawn from the APU equals the power flowing into the HV buses, where $N^{H V B}$ is the number of $\mathrm{HV}$ bus.

$$
P^{A P U}-\sum_{j} P_{j}^{A H V}=0, \forall j \in\left\{1, \ldots, N^{H V B}\right\}
$$

For all HV bus nodes, the power balance equations with the consideration of the power losses during the conversion are presented in (2). The input power for each HV bus includes the power from the connected generator/APU bus, and the converters when in boost mode, and the output power flows to the connected HV loads and the converters when in buck mode. The transmission efficiency of the cables $\epsilon_{j c}^{H V C}$ is introduced to present the cable losses. For all LV bus nodes, the power balance equations with transmission efficiency can be similarly represented in (3), where $N^{H L B}$ is the number of LV bus. The net power obtained from the converters and the adjacent LV buses should meet the load demand.

$$
\begin{gathered}
P_{j}^{G}+P_{j}^{A H V}+\sum_{c}\left(\epsilon_{j c}^{H V C} P_{c j}^{\prime H V C}-P_{j c}^{H V C}\right)-\sum_{p} P_{p}^{H V L}=0, \forall j \in\left\{1, \ldots, N^{H V B}\right\} \\
\sum_{c}\left(\epsilon_{c k}^{L V C} P_{c k}^{L V C}-P_{c k}^{L V C}\right)-\sum_{q} P_{q}^{L V L}-\sum_{k \neq k^{\prime}}\left(P_{k k^{\prime}}^{L V}-\epsilon_{k k^{\prime}}^{L V} P_{k^{\prime} k}^{L V}\right)=0, \forall k \in\left\{1, \ldots, N^{L V B}\right\}
\end{gathered}
$$

When the converter operates in buck mode, the power flows from the HV side to the LV side, the power flows in the opposite direction when the converter operates in buck mode. Therefore, for all DC/DC converter nodes, the power balance equation with the consideration of converter efficiencies and cable losses are presented in (1), where $N^{C}$ is the number of converters. 


$$
\left(\eta_{c} \sum_{j} P_{j c_{-} i n}^{H V C}-\sum_{j} P_{c j}^{\prime H V C}\right)-\left(\sum_{k} P_{c k}^{L V C}-\eta^{\prime}{ }_{c} \sum_{k} P_{c k \_i n}^{\prime L V C}\right)=0, \forall c \in\left\{1, \ldots, N^{C}\right\}
$$

Where

$$
\begin{gathered}
P_{j c i n}^{H V C}=P_{j c}^{H V C} \epsilon_{j c}^{H V C} \\
P_{c k_{-} i n}^{\prime L C}=P_{c k}^{\prime L C} \epsilon_{c k}^{L V C}
\end{gathered}
$$

\section{B. Power capacity constraints}

The power generated by the generator and transferred by the converters and cables should not exceed their power capacities for safety operation. In addition the power flow in each connection/direction depends on its connection status/direction indicator, when the connection status/direction indicator is 0 , the power flow through this connection line or in this direction is restricted to 0 . The group of these power upper and lower bounds constraints can be represented as the matrix in (5), where $\boldsymbol{P}=\left[P_{i}^{G}, P^{A P U}, P_{j}^{A H V}, P_{j c_{i n}}^{H V C}, P_{c k}^{L V C}, P_{c k \_i n}^{\prime L V C}, P_{j c}^{\prime H V C}, P_{k k^{\prime}}^{L V}\right]^{T}$ is the vector of decision variables for power flows in each connection/direction. $\boldsymbol{S}=\left[\alpha_{i}^{G}, \alpha^{A P U}, x_{j}, f_{j c}, f_{c k}, f_{c k}^{\prime}, f_{j c}^{\prime}, f_{k k^{\prime}}^{L V}\right]^{T}$ is the vector of binary variables representing the connection status or the direction indicator values. $\boldsymbol{P}_{\max }=$ $\left[P_{i_{-} \max }^{G}, P_{\max }^{A P U}, P_{\max }^{A P U}, P_{\max }^{C}, P_{\max }^{C}, P_{\max }^{C}, P_{\max }^{C}, P_{k k^{\prime} \_ \text {max }}^{L V}\right]^{T}$ is the vector of the power capacity corresponding to $\boldsymbol{P}$.

$$
\mathbf{0} \leq \boldsymbol{P} \leq \boldsymbol{S} \times \boldsymbol{P}_{\max }, \boldsymbol{S} \in \mathbb{B}
$$

\section{Unidirectional constraints}

In Fig. 2, the red lines represent the connections where the power has two optional flowing directions, but the power cannot flow in both directions simultaneously. For example, each converter can operate in either buck mode or boost mode, but not in both modes simultaneously. For an interconnection of LV bus node $k$ and $k^{\prime}$, the power could flow either from $k$ to $k^{\prime}$, or from $k^{\prime}$ to $k$. Therefore, the unidirectional constraints are set to avoid the conflicts in the power flow directions as represented in (6) - (8).

$$
\begin{gathered}
f_{j c}+f_{j c}^{\prime} \leq \beta_{j c} \\
f_{c k}+f_{c k}^{\prime} \leq \theta_{c k} \\
f_{k k^{\prime}}^{L V}+f_{k^{\prime} k}^{L V} \leq y_{k k^{\prime}}
\end{gathered}
$$

\section{Physical connection constraints}

The power contactors should be connected properly during the system operation to provide the system with the all possible operation topologies and prevent unexpected connection conditions. The APU connects to APU bus only when main generator failure occurs as presented in (9), and (10) - (11) indicate that each DC/DC converter cannot be connected to more than one HV and LV bus.

$$
\begin{gathered}
\alpha^{A P U}+\sum_{i} \alpha_{i}^{G} \leq N^{G} \\
0 \leq \sum_{j} \beta_{j c} \leq 1, \forall c \in\left\{1, \ldots, N^{C}\right\} \\
0 \leq \sum_{k} \theta_{c k} \leq 1, \quad \forall c \in\left\{1, \ldots, N^{C}\right\}
\end{gathered}
$$

\section{E. Optimal power allocation problem}

The objective of optimal power allocation is to minimise the total power losses from the generators to the loads. In different flight stages, the loads change, thus the optimal EPS configures power contactors on/off status, and the power flowing through each components to achieve a more efficient topology to reduce total power demand from generators. Therefore, the objective function for this problem formulation can be expressed as (12), and the constraints have been presented in (1)-(11).

$$
f(P)=\frac{1}{N^{G}+1}\left(\sum_{i}^{N^{G}} P_{i}^{G}+P^{A P U}\right)
$$

\section{F. Optimal generator sizing problem}


The objective of the optimal generator sizing problem is to minimise the maximum power required from each generator so that the overload requirements for each generator can be reduced. As the energy storage system is not considered in this paper, the optimisation should guarantee the generators can provide all demanded power including the load requirements and transmission losses during entire flight. Therefore, the cost function for this problem can be represented as (13) with the introduction of the new decision variable $P_{c a p}^{G}$ to represent the maximum power required from each generator.

$$
f(P)=\frac{1}{P_{\max }^{G}} \sum_{N} P_{i}^{G}+\frac{P_{\text {cap }}^{G}}{P_{\max }^{G}}
$$

The optimisation in this problem should be subject to the constraints introduced above in (1)-(11), besides, the additional constraints represented in (14) are proposed to indicate that the power supplied by each generator should not exceed the maximum needed power $P_{c a p}^{G}$, which has a predefined upper bound $P_{\max }^{G}$.

$$
\begin{gathered}
0 \leq P_{i}^{G} \leq \alpha_{i}^{G} P_{\max }^{G} \\
0 \leq P_{i}^{G} \leq P_{\text {cap }}^{G} \\
0 \leq P_{\text {cap }}^{G} \leq P_{\text {max }}^{G}
\end{gathered}
$$

\section{Linearization of the Model}

The aforementioned models for optimal power allocation and generator sizing problems in Section IV can be solved by the CPLEX solver if they only contain linear functions in the constraints and objectives. In the model constraints (2)-(1), the transmission efficiencies of the cables $\epsilon$ are assumed to be constant parameters based on the assumption of low cable losses to simplify the model. However, the DC/DC converter efficiency $\eta$ in (1) has a typical nonlinear relationship with regard to the input power as shown in Fig. 3, which can be represented in (15) to show the relationship in both buck and boost operation modes. In this case, the constraint function of DC/DC converter nodes power balance (1) can be presented in the format of (16). This will lead to a nonlinear problem unsuitable for MILP. Therefore, in this section, a piecewise function is proposed to linearize this nonlinear relationship.

$$
\begin{aligned}
& \eta_{c}=f\left(\sum_{j} P_{j c_{-} i n}^{H V C}\right) \\
& \eta_{c}^{\prime}=f\left(\sum_{k} P_{c k}^{\prime L V C} \text { in }\right) \\
& \left(f\left(\sum_{j} P_{j c_{-} i n}^{H V C}\right) \cdot \sum_{j} P_{j c_{\_} i n}^{H V C}-\sum_{j} P_{c j}^{\prime H V C}\right)-\left(\sum_{k} P_{c k}^{L V C}-f\left(\sum_{k} P_{c k \_i n}^{L V C}\right) \cdot \sum_{k} P_{c k \_i n}^{\prime L V C}\right)=0
\end{aligned}
$$

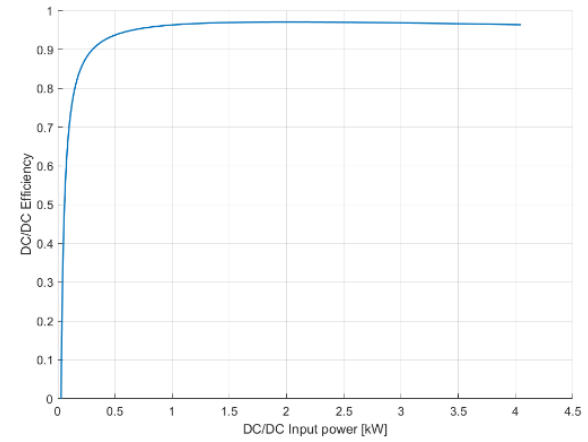

Fig. 3 A typical DC/DC converter efficiency curve dependent on input power

Instead of formulating the nonlinear efficiency curve in (16), a new decision variable $P_{C_{-} \text {out }}^{H V C}$ is introduced to represent the converter's output power in buck mode, and $P_{c_{-} \text {out }}^{{ }^{H V C}}$ for the output power in boost mode. Taking the variable $P_{C_{-} \text {out }}^{H V C}$ as an example, it can be approximated by a piecewise function, and each segment is a linear function relating to the input power as demonstrated in (17). Fig. 4(a) illustrates this procedure with the piecewise linear approximation of $P_{c_{-} \text {out }}^{H V C}$ by 5 linear segments. To verify the accuracy of the approximation, the approximated converter efficiency $\widetilde{\eta_{c}}$ can be derived in (18), and the curves are compared in Fig. 4 (b). The figure shows that the approximated efficiency $\widetilde{\eta_{c}}$ is very close to the typical efficiency curve. 


$$
\begin{aligned}
& P_{c_{-} \text {out }}^{H V C}=\eta_{c} \sum_{j} P_{j c_{-} \text {in }}^{H V C} \approx f_{p}\left(\sum_{j} P_{j c_{-} \text {in }}^{H V C}\right)\left\{\begin{array}{c}
k_{1} \sum_{j} P_{j c_{-} \text {in }}^{H V C}+b_{1}\left(m_{0} \leq \sum_{j} P_{j c_{\text {_in }}}^{H V C} \leq m_{1}\right) \\
k_{2} \sum_{j} P_{j c_{-} \text {in }}^{H V C}+b_{2}\left(m_{1} \leq \sum_{j} P_{j c_{\text {in }}}^{H V C} \leq m_{2}\right) \\
k_{3} \sum_{j} P_{j c_{-} \text {in }}^{H V C}+b_{3}\left(m_{2} \leq \sum_{j} P_{j c_{-} \text {in }}^{H V C} \leq m_{3}\right) \\
\vdots \\
k_{n} \sum_{j} P_{j c_{-} \text {in }}^{H V C}+b_{n}\left(m_{n-1} \leq \sum_{j} P_{j c_{-} \text {in }}^{H V C} \leq m_{n}\right)
\end{array}\right. \\
& \tilde{\eta_{c}}=\frac{k_{m} P_{c_{i n}}^{H V C}+b_{m}}{P_{c i n}^{H V C}}=k_{m}+\frac{b_{m}}{P_{c i n}^{H V C}}
\end{aligned}
$$

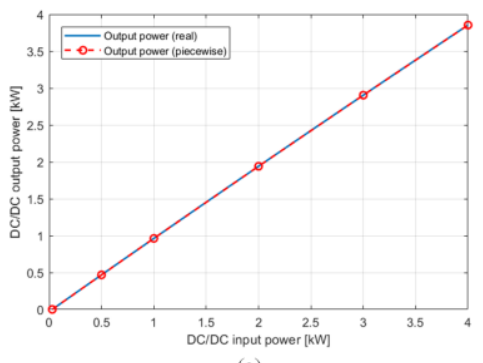

(a)

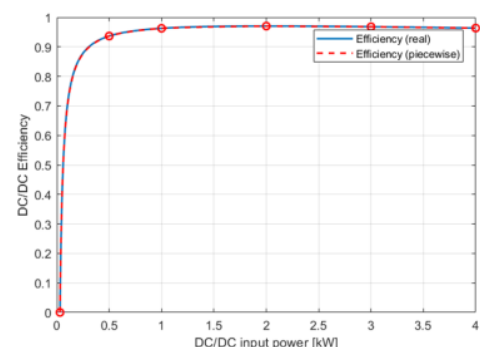

(b)

Fig. 4 Piecewise curves (a) Piecewise output power curve compared to the output power based on typical efficiency curve (b) The piecewise function derived efficiency curve compared to the typical efficiency curve

\section{Simulation Results}

In this section, the proposed mathematical models and linearization methodology in Section IV and V are applied to the optimal power allocation and generator sizing problems for the novel HVDC MPC EPS in Fig. 1. The problems are simulated and solved by CPLEX.

Two case studies are provided to show the power scheduling results of the optimal power allocation with different load requirements and generator fault situations. In Case 1, the HV loads are $\{40,40\} \mathrm{kW}$, and the LV loads are $\{1$, $2,2,1\} \mathrm{kW}$, and both generators are defined as normal operation mode. In Case 2, generator 2 is assumed to be failing to operate, and the HV loads are $\{40,30\} \mathrm{kW}$, while the $\mathrm{LV}$ loads are $\{2,3,6,4\} \mathrm{kW}$. The power routing which results in the OPA for the EPS are illustrated in Fig. 5 and Fig. 6 for the two cases respectively. From the results in Fig. 5 and Fig. 6, it can be observed that the power balance is realised for all the nodes and the most efficient connections are achieved as expected. In addition, all the power converters are working in high efficiency operation range and located well on the real efficiency curve. This indicates that the optimal power allocation has been realised to reduce the total transmission power losses by the proposed optimisation method. Fig. 6 also shows that the APU can be automatically connected to the HV bus when one generator fails, which shows the model capability to cope with generator failure cases.
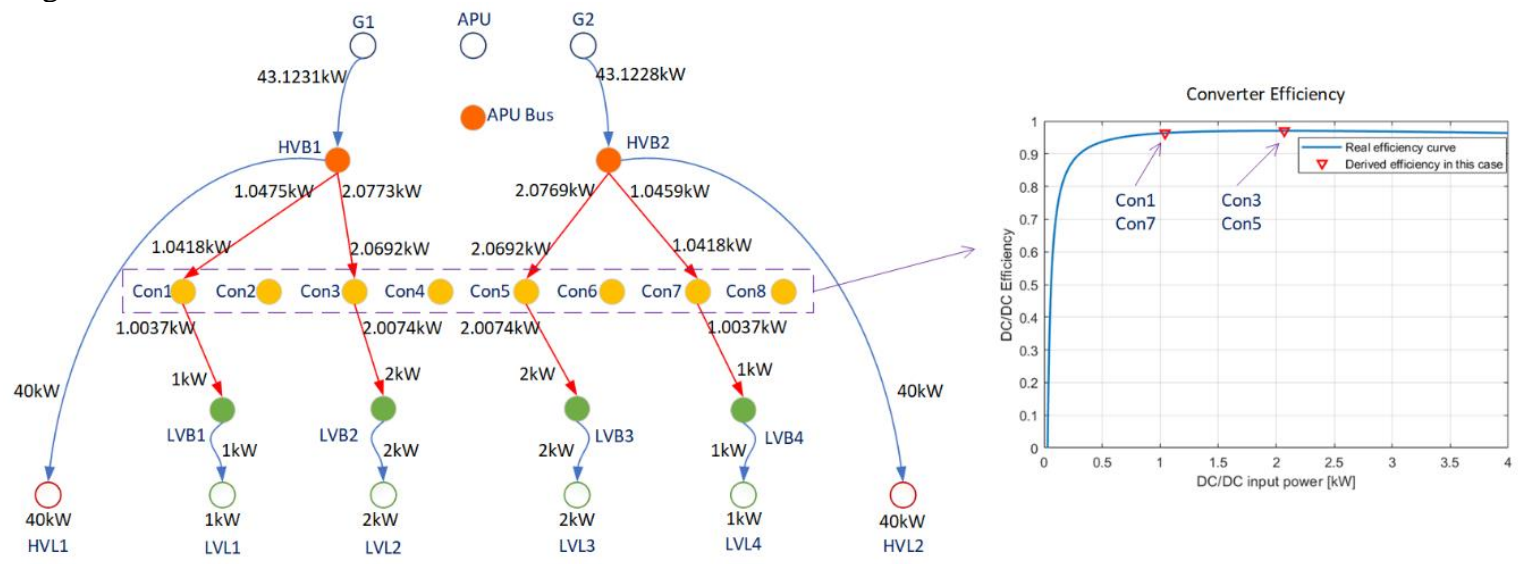

Fig. 5 Power routing results to have the optimal power allocation in Case 1 


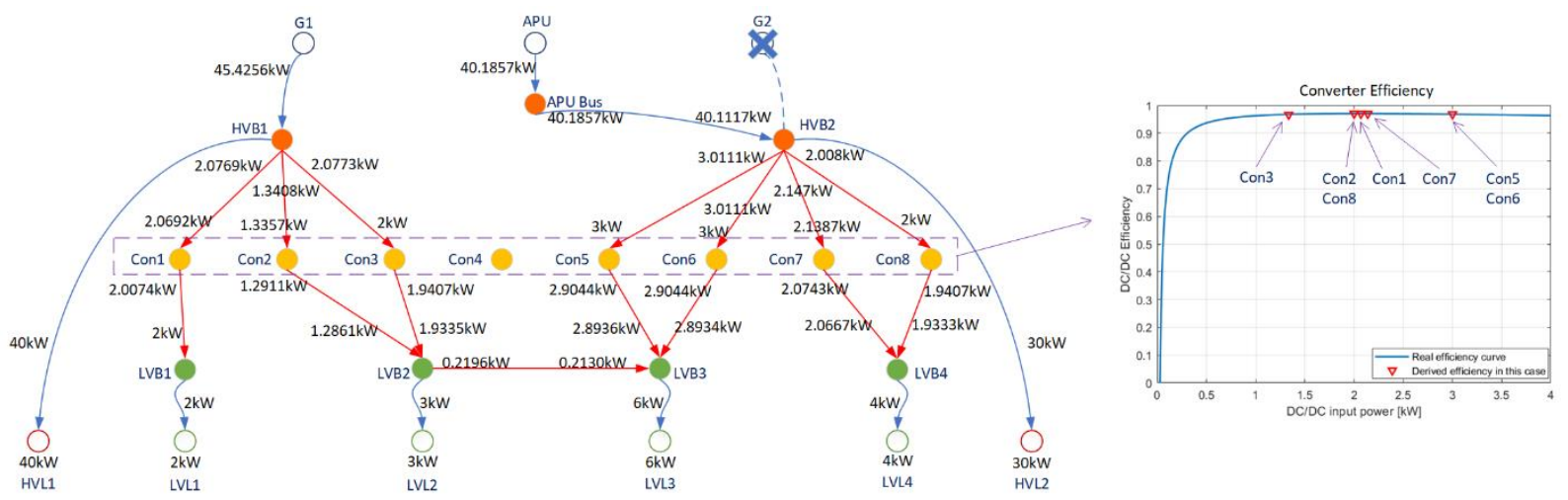

Fig. 6 Power routing results to have the optimal power allocation in Case 2

One typical flight cycle has eight stages including taxiing to the runway, take-off, climbing, cruise, descent, approach, landing, and taxiing to the terminal. It is assumed that during the whole flight stage the loads varies. The optimal power allocation for the two generators in different flight stages is presented in Fig. 7 (a), and Fig. 7 (b) shows the optimal generator sizing results regarding the load requirement changes in eight stages. Comparing the two figures, the generators work in a more balanced manner in the optimal generator sizing problem, because the formulation of this problem has an additional objective to reduce the power requirement of each generator rather than only reducing the total power needs.
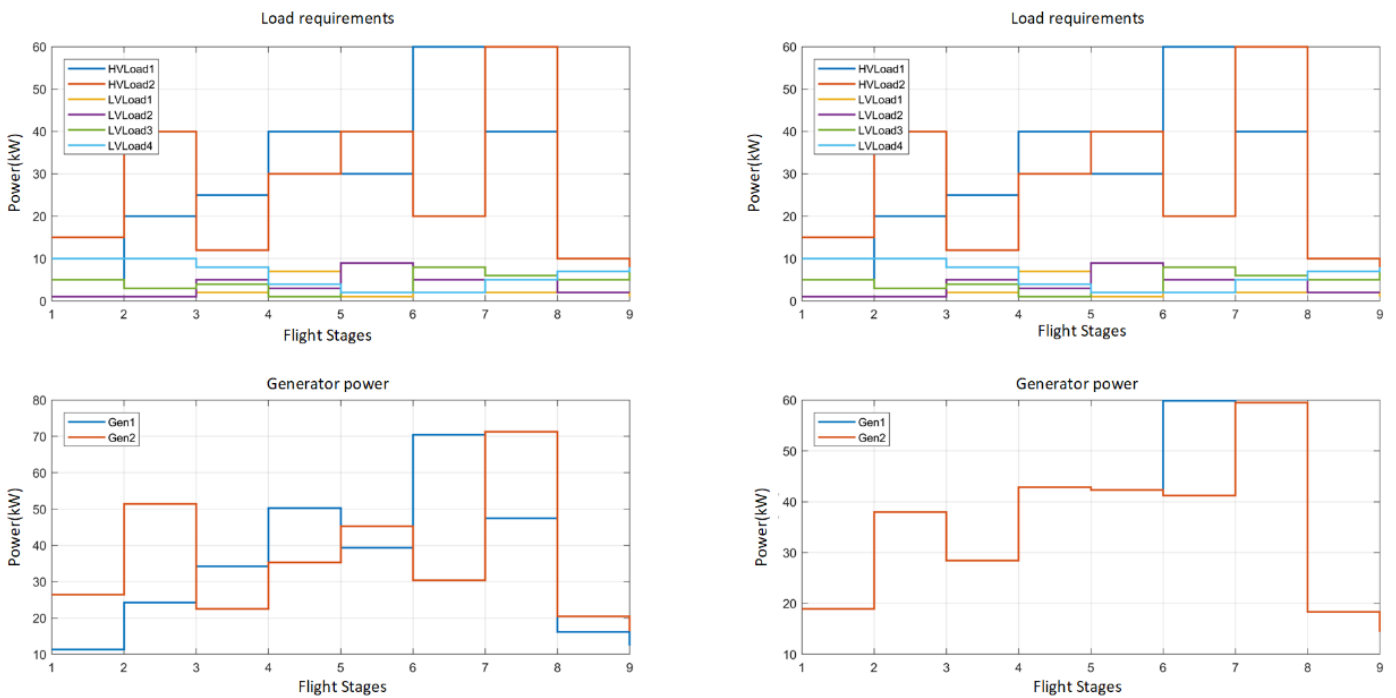

(b)

Fig. 7 Generator power in different flight stages (a) simulation results of optimal power allocation (b) simulation results of optimal generator sizing

\section{Conclusion}

This paper formulates a mathematical model for both OPA and OGS problems of a MPC HVD EPS architecture to reduce the total power needs from the power sources and minimise the required overload capabilities for each generator. A formulation method based on optimal power flow (OPF) model is proposed to constitute the system constraints of power balancing and limitations for each bus/converter, the bus/contactor connection logic, the transmission power losses in the cables and the nonlinear converter efficiency characteristics. By using the piecewise linearization techniques, the proposed model can be solved by MILP to reduce the computational complexity. Simulation results have shown that the optimisation model and the approach can achieve good performance and the optimal solutions can be obtained for different flight stages. 


\section{Appendix}

The transmission efficiency of the cables used in the simulation are listed in the following tables.

Table 1 Transmission efficiencies of buses interconnections

\begin{tabular}{c|c}
\hline Parameters & Values \\
\hline$\epsilon_{j}^{A H V}$ & 0.9998 \\
\hline$\epsilon_{k k^{\prime}}^{L V}$ & 0.9000 \\
\hline
\end{tabular}

Table 2 Transmission efficiencies of the cables between HV/LV buses and DC/DC converters

\begin{tabular}{c|c|c|c|c|c|c|c|c}
\hline \multicolumn{7}{c}{ Transmission efficiencies $\epsilon_{j c}^{H V C}$ between HV bus $j$ and DC/DC converters $c$} \\
\hline$\epsilon_{j c}^{H V C}$ & DC/DC 1 & DC/DC 2 & DC/DC 3 & DC/DC 4 & DC/DC 5 & DC/DC 6 & DC/DC 7 & DC/DC 8 \\
\hline HV bus 1 & 0.9963 & 0.9962 & 0.9961 & 0.9960 & 0.983 & 0.982 & 0.981 & 0.980 \\
\hline HV bus 2 & 0.980 & 0.981 & 0.982 & 0.983 & 0.9963 & 0.9962 & 0.9961 & 0.9960 \\
\hline \multicolumn{7}{|c|}{ Transmission efficiencies $\epsilon_{c k}^{L V C}$ between DC/DC converter $c$ and LV bus $k$} \\
\hline$\epsilon_{c k}^{L V C}$ & DC/DC 1 & DC/DC 2 & DC/DC 3 & DC/DC 4 & DC/DC 5 & DC/DC 6 & DC/DC 7 & DC/DC 8 \\
\hline LV bus 1 & 0.9963 & 0.9962 & 0.9961 & 0.9960 & 0.983 & 0.982 & 0.981 & 0.980 \\
\hline LV bus 2 & 0.9960 & 0.9961 & 0.9963 & 0.9962 & 0.9835 & 0.9825 & 0.9815 & 0.9805 \\
\hline LV bus 3 & 0.9805 & 0.9815 & 0.9825 & 0.9835 & 0.9963 & 0.9962 & 0.9961 & 0.9960 \\
\hline LV bus 4 & 0.980 & 0.981 & 0.982 & 0.983 & 0.9960 & 0.9961 & 0.9963 & 0.9962 \\
\hline
\end{tabular}

\section{Acknowledgments}

This work is funded by the INNOVATIVE doctoral programme. The INNOVATIVE programme is partially funded by the Marie Curie Initial Training Networks (ITN) action, and partially by the Institute for Aerospace Technology (IAT) at the University of Nottingham.

\section{References}

[1] B. H. Nya, J. Brombach, and D. Schulz, "Benefits of higher voltage levels in aircraft electrical power systems," Electr. Syst. Aircraft, Railw. Sh. Propulsion, ESARS, 2012.

[2] B. Sarlioglu and C. T. Morris, "More Electric Aircraft: Review, Challenges, and Opportunities for Commercial Transport Aircraft," IEEE Trans. Transp. Electrif., vol. 1, no. 1, pp. 54-64, 2015.

[3] R. T. Naayagi, "A review of more electric aircraft technology," 2013 Int. Conf. Energy Effic. Technol. Sustain. ICEETS 2013, pp. 750-753, 2013.

[4] J. C. Swierczek, F. Mollet, C. Saudemont, R. Meuret, and B. Robyns, "Power management of a regenerative local HVDC aircraft network using supercapacitors," 15th Int. Power Electron. Motion Control Conf. Expo. EPE-PEMC 2012 ECCE Eur., pp. 1-8, 2012.

[5] X. Roboam, "New trends and challenges of electrical networks embedded in "“more electrical aircraft,"” 2011 IEEE Int. Symp. Ind. Electron., pp. 26-31, 2011.

[6] M. Maasoumy, P. Nuzzo, F. Iandola, M. Kamgarpour, A. Sangiovanni-Vincentelli, and C. J. Tomlin, "Optimal load management system for Aircraft Electric Power distribution,” IEEE Conf. Decis. Control, pp. 2939-2945, 2013.

[7] A. Barzegar, R. Su, C. Wen, L. Rajabpour, Y. Zhang, and M. Y. Lee, "Intelligent Power Allocation and Load Management of More Electric Aircraft," Ieee Peds, no. June, pp. 533-538, 2015.

[8] Y. Zhang, R. Su, C. Wen, M. Y. Lee, and C. Gajanayake, "Distributed power allocation and scheduling for electrical power system in more electric aircraft," IECON 2016 - 42nd Annu. Conf. IEEE Ind. Electron. Soc., pp. 102-107, 2016.

[9] Y. Zhang, G. Ooi, and H. Peng, "Design of the Power Management System for Electrical Power Systems in More Electric Engine / Aircraft," no. 3, pp. 0-5, 2018.

[10] Y. Zhang et al., "An Energy Efficient Power Management Solution for a Fault-tolerant More Electric Engine/Aircraft," IEEE Trans. Ind. Electron., vol. PP, no. c, pp. 1-1, 2018.

[11] M. H. Moradi and M. Abedini, "Electrical Power and Energy Systems A combination of genetic algorithm and particle swarm optimization for optimal DG location and sizing in distribution systems," Int. J. Electr. Power Energy Syst., vol. 34, no. 1 , pp. 66-74, 2012. 
[12] P. Thota and M. Venkata Kirthiga, "Optimal siting \& sizing of distributed generators in micro-grids," 2012 Annu. IEEE India Conf. INDICON 2012, pp. 730-735, 2012. 